\title{
The Egyptain 1.88-m Telescope
}

\author{
Anas M. I. Osman
}

National Research Institute of Astronomy and Geophysics, Helwan, Egypt,amiosman46@hotmail.com

\begin{abstract}
The Kottamia 1.88-m reflecting telescope in Egypt is the largest in the Middle East and North Africa. An extensive upgrading programe has been undertaken for this telescope to increase its efficiency. A new Zerodur optical system has been delivered by Carl Zeiss, Germany, and a new CCD system including an acquisition Camera Tek 1024 x 1024 pixels, with pixel size $24 \times 24$ micron and LN cooling, and an offset guiding camera Kodak $1080 \times 1024$ pixels with pixel size $16 \times 16$ micron and thermoelectric cooling. This CCD system has been attached to the Newtonian focus for direct imaging with scale $22.5 \operatorname{arcsec} \mathrm{mm}^{-1}$. The aluminizing plant has also been refurbished by Balzer, the new pumping system can accommodate mirrors up to $2 \mathrm{~m}$. The unit is supplied with a microprocessor, which controls and checks all recoating steps, closes all valves and stops the operation in any emergency. A Cassegrain spectrograph (donation from Okayama Astrophysical Observatory, Japan) will be attached to the telescope, after modification, for use with a CCD camera instead of the image-intensifier used before. This spectrograph will be used to obtain medium-dispersion and low dispersion spectra for faint stars and galaxies down to 15 th magnitude.
\end{abstract}

\section{Introduction}

Astronomy in Egypt is an old science. The first reasonable-sized telescope used for astronomical observations was the $0.9-\mathrm{m}$ reflector at Helwan Observatory (south of Cairo), which has been used for many decades since 1905 to observe many celestial objects, specially in the declination zone $0^{\circ}$ to $30^{\circ}$, and many new objects have been detected. More than 1907 nebulae were observed in that zone and, among them, 505 nebulae were new discoveries. In addition, observations of many comets (e.g. Halley 1910) and planets with their satellites have been collected. In 1962 a new era of astronomy began in Egypt with the construction of the $1.88-\mathrm{m}$ reflecting telescope at Kottamia $(70 \mathrm{~km}$ due east from Cairo, in the eastern desert of Egypt), see Figure 1. It is constructed on a plateau at about $500 \mathrm{~m}$ above see level with geographical coordinates $\phi=29^{\circ} 55.9^{\prime} \mathrm{N}, \lambda=$ $31^{\circ} 49.5^{\prime} \mathrm{E}$. The telescope was manufactured by Grubb Parsons of England and it can be used in the Newtonian $(f / 4.9)$, Cassegrain $(f / 18)$ and coudé $(f / 28.9)$ focii with scales of $22.5,6$ and 3.8 arcsec $\mathrm{mm}^{-1}$ respectively. This telescope was provided with: a Newtonian camera $(f / 4.9)$ for direct imaging with correcting lens (field of view of about one degree); a Cassegrain spectrograph with two 
cameras of dispersions of $100 \mathrm{~A} \mathrm{~mm}^{-1}$ and $48 \mathrm{~A} \mathrm{~mm}^{-1}$ at $4800 \mathrm{~A}$; and a twochannel photoelectric photometer for stellar photometry with photon counter.

Since the telescope started functioning in 1964, hundreds of observations have been secured for stars, galaxies, star clusters, planets, comets, etc. by Egyptian and foreign astronomers. Based on these observations, a large number of papers have been published and tens of M. Sc. and Ph.D. degrees have been awarded. Due to the successive re-aluminizing of the telescope mirror and the chemical treatment required for the process during about 30 years of work, a serious problem with the surface of the main mirror was detected. The mirror surface would not accept any recoating properly and the resultant coating was dim, poorly reflecting and short-lived. Many attempts were made to clean the surface and to recoat it, without success. Several reports concluded that repeated cleaning of the mirror surface with chemicals had leached it. On the other hand, the detectors provided with the telescope became old and needed to be replaced by new sophisticated ones. After evaluation of the situation, an extensive program for upgrading the telescope was undertaken to provide the telescope with:

- a new Zerodur optical system (primary and Cassegrain mirrors),

- a new large-format CCD camera system with liquid-nitrogen cooling and a CCD camera for offset guiding with thermoelectric cooling,

- a new fast grating spectrograph,

- an updated aluminizing plant for the Kottamia Observatory,

- upgraded the computing facilities.

\section{Upgrading Program for the Kottamia 1.88-m Telescope}

\subsection{CCD Camera System}

In 1992, the upgrading program was begun by a contract with Astro Cam (Pix Cellent now), U.K., to provide the telescope with a CCD camera system for scientific observations and for guiding purposes, with the following specifications:

A - The Scientific Camera: This is to be used mainly for direct imaging and provided with LN cooling. The main chip is Tektronix with format 1024 x 1024 pixel with pixel size $24 \times 24$ microns square, thinned back-illuminated with an anti-reflection coating, to enhance the the chip sensitivity to shorter wavelengths. It is characterized by high dynamic range, excellent charge-transfer rate at low signal level with low dark current and maximum readout flexibility. The quantum efficiency reaches its maximum value $(80 \%)$ at room temperatrue between $6000 \mathrm{~A}$ and $7000 \mathrm{~A}$.

B - The Guiding Camera: This camera is to be used for guiding during observations. The main chip is Kodak KAF-1300 with format of $1280 \times 1024$ pixels with pixel size $16 \times 16$ microns square, cooled thermoelectrically. Its spectral response is from blue to near infrared spectral range with maximum quantum efficiency $(\sim 50 \%)$ at $8000 \mathrm{~A}$. A long pass-band filter RG 610 is installed at the 


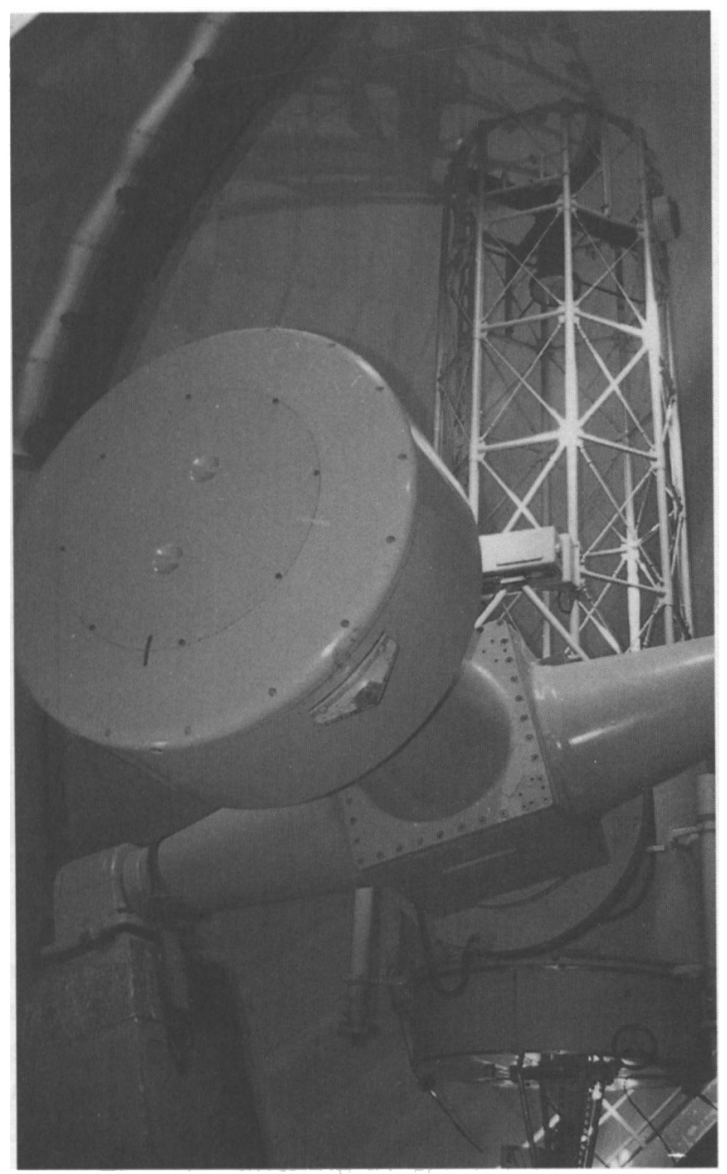

Figure 1. The Kottamia 1.88-m Telescope, Egypt

front of the camera to improve the $\mathrm{S} / \mathrm{N}$ ratio. The two cameras are controlled by the DDE 4202 controller unit and Imager 2 software package. They have been tested and mounted at the Newtonian focus of the telescope $(f / 4.9$, plate scale $=22.53$ arcsec $\mathrm{mm}^{-1}$ ) for direct imaging. This system can be also used at the Cassegrain focus of the telescope $\left(f / 18\right.$, plate scale $\left.=6 \operatorname{arcsec} \mathrm{mm}^{-1}\right)$ although the long tube of the telescope can affect the optical path to the Cassegrain focus by some disturbance.

\subsection{New Optical system for the Kottamia 1.88-m telescope}

In 1994 a contract with Zeiss Jena (Germany) was signed to provide the Kottamia 1.88-m telescope with a new optical system (primary mirror and Cassegrain secondary) made of Zerodur, together with modification of the main-mirror cell and the Cassegrain cell to suit the new optical system. A new design for the supporting system of the main mirror was produced by Zeiss, using the old metal cell of the Kottamia telescope. It consists of 18 axial supports distributed in two rings, the inner ring with six supports and the outer with 12, see Figure 2. 


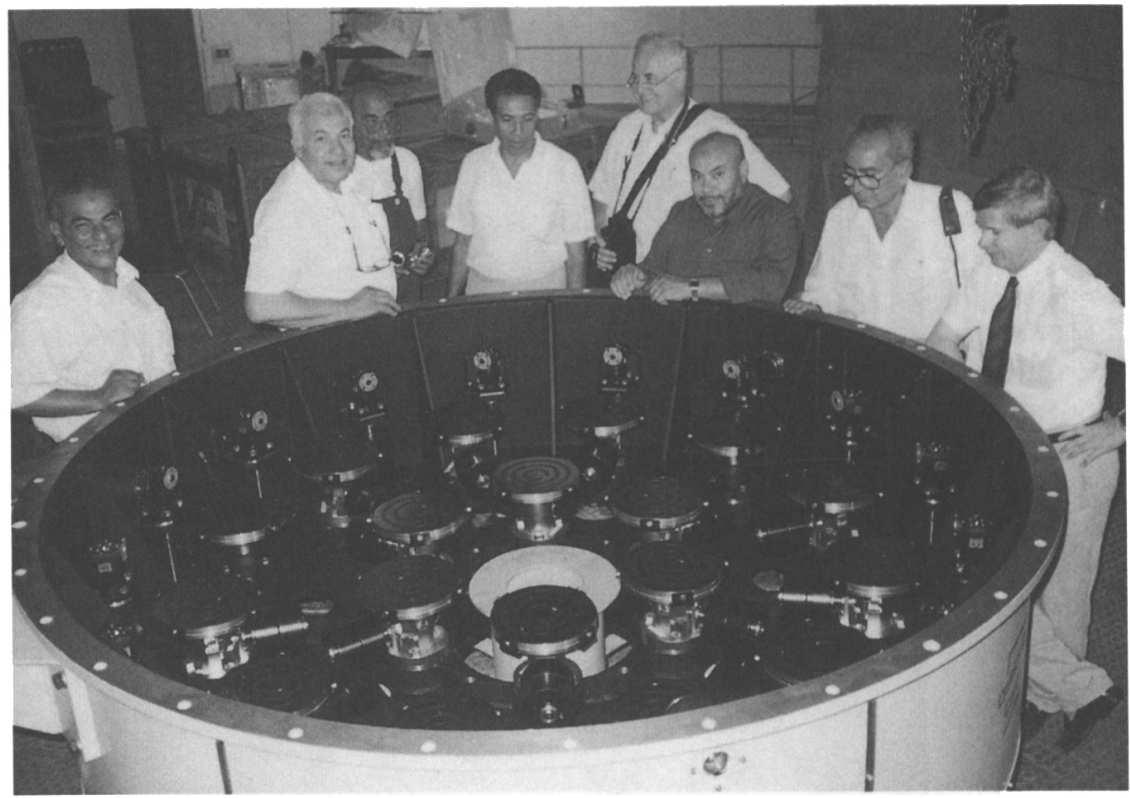

Figure 2. Distribution of the 18 axial supports in the modified cell

The mirror will be supported by 16 lateral Zerodur pads cemented at the mirror edge, see Figure 3. These pads are fixed on 16 radial counterweight-support units, which are fixed on the inner edge of the mirror cell. The radial position of the mirror in the cell is defined by three units (three fixation points), two of them are hard points (fixed, adjustable stops) and the third point gives a soft pressure to the mirror edge by the use of a spring.

The new primary mirror has the specifcations shown in Table 1.

Table 1. The New Primary Mirror Specification

\begin{tabular}{ll}
\hline \hline Focal length & $9138 \mathrm{~mm}$ \\
Weight & $1600 \mathrm{~kg}$ \\
Material density & $2.53 \times 10^{-3} \mathrm{~kg} \mathrm{~mm}^{-3}$ \\
Radius of the mirror & $965 \mathrm{~mm}$ \\
Radius of inner hole & $94 \mathrm{~mm}$ \\
Diameter of encircled $80 \%$ of energy & $\leq 0.35$ arcsec \\
\hline
\end{tabular}

The new optical system and the modified cell arrived at the site during the summer of 1997. Acceptance tests on site were begun in April 1998, by Zeiss experts, and the results were reasonable but not sufficient. Another test campaign was begun by Zeiss experts on 17 June 1998 and, after few nights, they found that the results were changeable and unsatisfactory. The primary-mirror supporting system was examined and, after the dismantling the cell, Zeiss found that 12 out of the 16 radial glued pads were damaged and had come loose from 


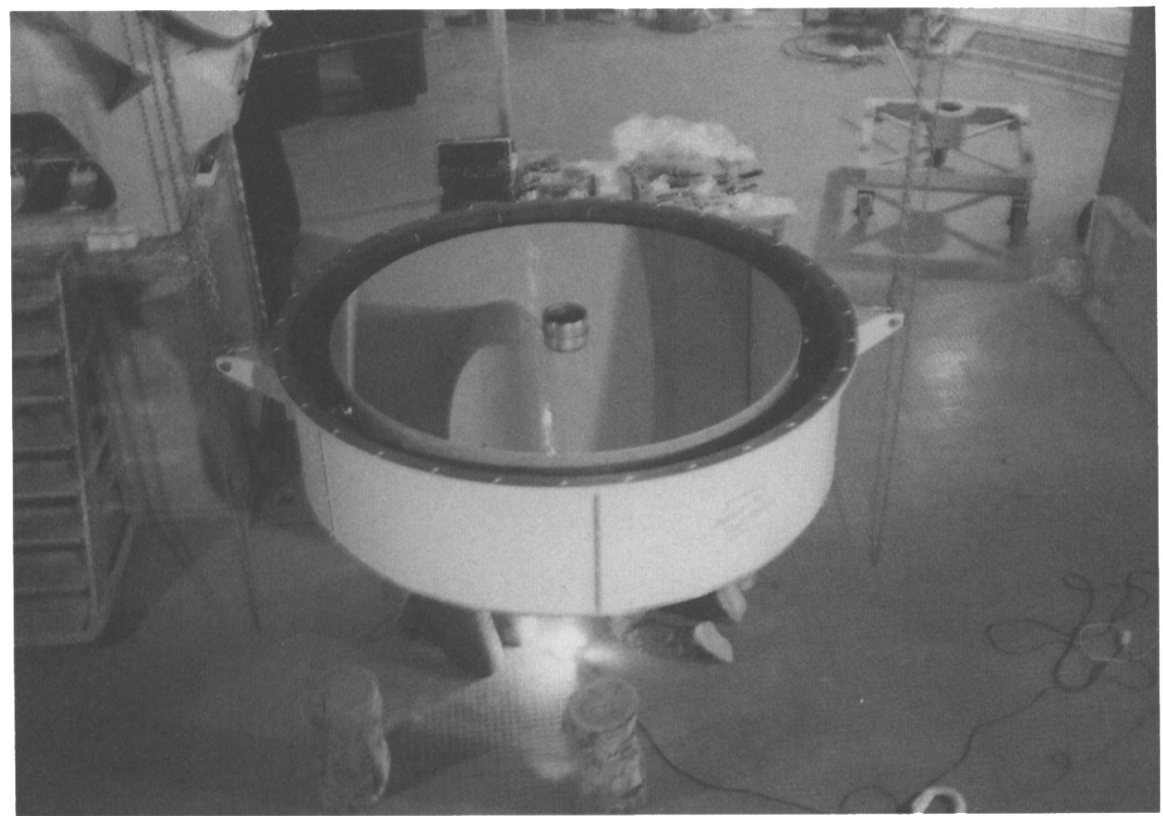

Figure 3. Installation of the new primary mirror in its modified cell

the body of the main mirror. The only explanation offered by Zeiss was that some glued supports fell off because of the material of the glue. After regluing these 12 pads, the Zeiss team began another test during November 1998 and took some frames and analyzed them to evaluate the Zernike Coefficients. The observations were very limited, covering a very short time during two nights (total observing time 6 hours) for a few stars around the zenith. Since this test was performed for a very short time and around the zenith, in the absence of any NRIAG consultant, Zeiss was asked to make the telescope available for more observing time to check the accuracy and stability of the whole system before acceptance. During one of the tests by Egyptian astronomers in June 1999, a hard sound of a sliding part below the main mirror was heard, observations were stopped and Zeiss was informed and asked to examine the mirror and the supporting system. After once more dismantling the mirror cell, Zeiss experts found that two lateral pads were loose again and separated from the mirror body (these two pads were among the twelve reglued pads separated before). The supporting system and its design became doubtful and Zeiss must find a solution to guarantee the reliability of the supporting system. In the technical proposal of their offer, Zeiss stated that the equilibrium of the mirror would be guaranteed in all positions of the telescope. From that time, the situation became complicated, since Zeiss insists that the main reason for the damage is tilting the telescope tube below horizon by $31^{\circ}$, although this could not have happened in any way. Accordingly, both sides have gone to arbitration to solve the problem and to finalize the project. 


\subsection{Updating the aluminizing plant of Kottamia Observatory}

The Kottamia aluminizing plant is used for coating the primary and secondary mirror of the telescope, see Figure 4. It can hold mirrors up to two meters in diameter. The unit was delivered by Edwards (England) about 30 years ago, and was used efficiently since that time. Since it was equipped with a semiautomatic pumping system with limited safety facilities, it was important for the safety of the new optical system to upgrade the present unit by replacing the pumping system with a new powerful, automatically controlled unit with accurate measuring facilities. This new pumping system is adapted to the old aluminizing chamber which is still in good condition. At the end of 1997 a contract was signed with Balzers to undertake the upgrading with the aims of:

1 controlling the high-vacuum system by a modern automatic control allowing all operational steps to be programmed,

2 giving the pumping system a high efficiency and equipping it with all necessary safety elements,

3 reducing the manual operations in order to avoid personal errors,

4 preventing back-streaming of diffusion-pump oil and accelerating the evacuation process,

5 providing a very high-speed pumping system for a short processing time,

6 protecting the mirror in case of any failure by safety and control units.

After providing and installing all parts of the new pumping system, the aluminizing plant has been tested for a vacuum of pressure of $10^{-6}$ mbar inside the chamber and the results were satisfactory.

\subsection{New Cassegrain spectrograph}

A Cassegrain grating spectrograph has been donated by Okayama Astrophysical Observatory, Japan, to Kottamia Observatory. It will be attached to the telescope after modifications for using a CCD camera instead of the image intensifier used before. This spectrograph covers the wavelength range $4000 \mathrm{~A}-8000$ A, with slit length of $15 \mathrm{~mm}$ ( $1.5 \mathrm{arcmin}$ on the sky). It will be used at middledispersion to low-dispersion for faint stars and galaxies down to around 15th magnitude. A new CCD camera head has been ordered from Pix Cellent company to be used with this spectrograph. It is an EEV CCD 42-10 CCD chip with $2048 \times 512$ pixels grade 1 with pixel size 13.5 microns square and an active image area of $27.6 \times 6.9 \mathrm{~mm}^{2}$, with $\mathrm{LN}$ cooling. The CCD is back illuminated (thinned) with an extended AR coating. Typical quantum efficiencies are $>90 \%$ at 500 $\mathrm{nm}, 50 \%$ at $350 \mathrm{~nm},>80 \%$ at $4000-6500 \mathrm{~A}$ and $30 \%$ at $9000 \mathrm{~A}$. The readout noise is three electrons RMS at low pixel rates. The active spectral range of the camera is from 200 to $1060 \mathrm{~nm}$ In addition, a CCD camera for tracking has been orderd, it includes a thermoelectric CCD head, of type TE/3 Kodak KAF V0040 grade 1 device with $768 \times 512$ pixels, with pixel size 9.0 microns square. The active image area is $6.8 \times 4.5 \mathrm{~mm}^{2}$. The CCD is front illuminated (thinned) with a typical QE of $35 \%$ at $600 \mathrm{~nm}$. Its readout noise is eight electrons RMS at low pixel rates. 


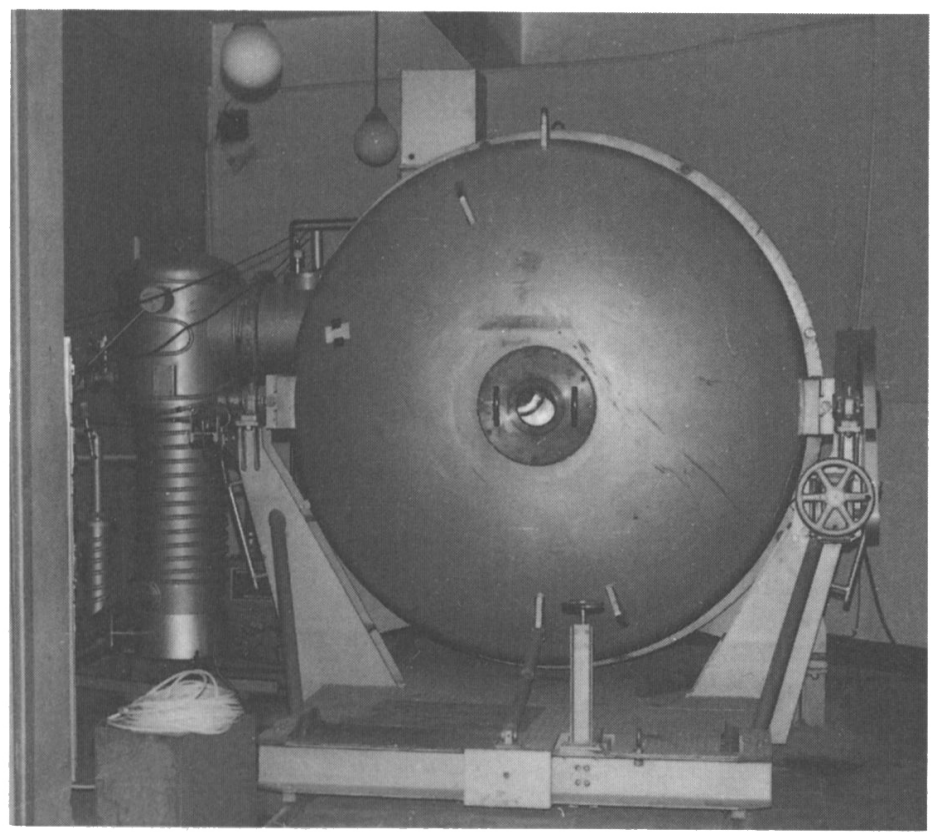

Figure 4. The Kottamia aluminizing plant after updating

\subsection{Upgrading the computing facilities}

During the last three years, the Kottamia observatory has been provided with a powerful computing facilities including two Sun Spark workstations and some Pentium PC computers. These systems are used in controlling the CCD systems of the telescope and analyzing the observation by different software packages.

The Kottamia 1.88-m telescope will be back in commission very soon after the problems of the supporting system have been solved. It is a good telescope and can serve all astronomers in the Middle East and North Africa since it is the largest telescope in the region. It needs all the support from all authorities to develop astronomy in many developing countries in the region especially after such upgrading project.

\section{References}

El Bassuny, A. 1998, African Skies, No. 2, p. 15.

Hanafy, D. and Heileman, W. 1999, African Skies, No. 4, p. 7.

Hassan, S.M. 1998, African Skies, No. 2, p. 16.

Samaha, A.H. 1964, Helwan Observatory Bulletin, No. 62. 


\section{Discussion}

In answer to Kochhar's query about the source of funds for upgrading the Kottamia telescope, Osman said that most of the funding came from the budget for the last five-year plan of the National Research Institute of Astronomy and Geophysics, Helwan-Cairo; part also came from the Egyptian Ministry of International Cooperation. In answer to Hingley, Osman said that the mirror supports are not active. Hingley also commented that the Kottamia telescope was one of about half-a-dozen of that size built by Grubbb-Parsons. He was glad to hear of the rebirth of this one and drew attention to a recent article on these telescopes by Richard Jarrell in the Journal for the History of Astronomy, (Vol. 30, pp. 359-90, 1999).

Al-Sabti commented that when he went to use the telescope some time ago, the lack of funds was obvious and there was no permanent staff at the Observatory. He was glad to hear that things were improving. Osman said that the financial situation is improving. They had $\$ 2.5 \mathrm{M}$ from the Egyptian government to upgrade the telescope and to maintain the Observatory. The only permanent staff at the Observatory are the technical workers. Astronomers visit Kottamia only to observe. There are now about 35 astronomers, with Ph.D. degrees, with permanent jobs at the Institute. 